\title{
CANONICAL DUAL FINITE ELEMENT METHOD FOR SOLVING NONCONVEX MECHANICS AND TOPOLOGY OPTIMISATION PROBLEMS
}

\author{
ELAF J. ALI ${ }^{\circledR}$
}

(Received 28 September 2019; first published online 25 November 2019)

\section{Mathematics subject classification: primary 90C46; secondary 90C26.}

Keywords and phrases: canonical duality theory, canonical penalty-duality, 3D topology optimisation problem, large deformation, postbuckling problems, nonconvex functional, finite element method.

Canonical duality theory (CDT) is a newly developed, potentially powerful methodological theory which can transfer general multiscale nonconvex/discrete problems in $\mathbb{R}^{n}$ to a unified convex dual problem in continuous space $\mathbb{R}^{m}$ with $m \leq n$ and without a duality gap. The associated triality theory provides extremality criteria for both global and local optimal solutions, which can be used to develop powerful algorithms for solving the general nonconvex variational problem.

First, this thesis presents a detailed study of large deformation problems in a twodimensional structural system. Based on canonical duality theory, a canonical dual finite element method is applied to find a global minimisation to the general nonconvex optimisation problem using a new primal-dual semidefinite programming algorithm. Applications are illustrated by numerical examples with different structural designs and different external loads.

Next, a new methodology and algorithm for solving post-buckling problems of a large deformed elastic beam is investigated. The total potential energy of this beam is a nonconvex functional, which can be used to model both pre- and post-buckling phenomena. By using the canonical dual finite element method, a new primal-dual semidefinite programming algorithm is presented, which can be used to obtain all possible post-buckled solutions. In order to verify the triality theory, mixed meshes of different dual stress interpolations are applied to obtain the closed dimensions between discretised displacement and discretised stress. Applications are illustrated by several numerical examples with different boundary conditions. We find that the global minimum solution of the nonconvex potential leads to a stable configuration of

Thesis submitted to Federation University in February 2018; degree approved on 25 September 2018; principal supervisor David Gao.

(C) 2019 Australian Mathematical Publishing Association Inc. 
the buckled beam, the local maximum solution leads to the unbuckled state and both of these solutions are numerically stable. However, the local minimum solution leads to an unstable buckled state, which is very sensitive to the external load, thickness of the beam, numerical precision and the size of finite elements.

Finally, a mathematically rigorous and computationally powerful method for solving three-dimensional topology optimisation problems is demonstrated. This method is based on CDT developed by Gao [4] in nonconvex mechanics and global optimisation. It shows that the so-called NP-hard knapsack problem in topology optimisation can be solved deterministically in polynomial-time via a canonical penalty-duality (CPD) method to obtain a precise global optimal 0-1 density distribution at each volume evolution. The relation between this CPD method and Gao's pure complementary energy principle is revealed for the first time. A CPD algorithm is proposed for three-dimensional topology optimisation of linear elastic structures. Its novelty is demonstrated by benchmark problems. Results show that, without using any artificial technique, the CPD method can provide mechanically sound optimal design and that it is much more powerful than the well-known bi-directional evolutionary structural optimization (BESO) and the solid isotropic material with penalization (SIMP) topology optimisation methods. In addition, computational complexity and conceptual/mathematical mistakes in topology optimisation modelling and popular methods are explicitly addressed. Some of the research from the thesis has been published in [1-5].

\section{References}

[1] E. J. Ali and D. Y. Gao, 'Canonical finite element method for solving nonconvex variational problems to post buckling beam problem', AIP Conf. Proc. 1776(1) (2016), Article ID 090045.

[2] E. J. Ali and D. Y. Gao, 'Improved canonical dual finite element method and algorithm for post buckling analysis of nonlinear Gao beam', in: Canonical Duality Theory: Unified Methodology for Multidisciplinary Study (Springer, Cham, 2017), 277-289.

[3] E. J. Ali and D. Y. Gao, 'On SDP method for solving canonical dual problem in post buckling of large deformed elastic beam', Commun. Math. Sci. 16(5) (2018), 1225-1240.

[4] D. Y. Gao, 'Canonical duality theory for topology optimization', in: Canonical Duality-Triality: Unified Theory and Methodology for Multidisciplinary Study (eds. D. Y. Gao, N. Ruan and V. Latorre) (Springer, New York, 2019), 263-276.

[5] D. Gao and E. J. Ali, 'A novel canonical duality theory for solving 3-D topology optimization problems', in: Emerging Trends in Applied Mathematics and High-Performance Computing (eds. V. K. Singh, D. Y. Gao and A. Fisher) (Springer, New York, 2019), 209-246.

ELAF J. ALI, Faculty of Science and Technology,

Centre for Informatics and Applied Optimisation,

Federation University Australia, Mt Helen,

Victoria 3353, Australia

and

Mathematics Department, College of Science,

University of Basrah, Basra, Iraq

e-mail: elaf.j.ali@gmail.com 